\title{
Étude des relations entre la précocité du tournesol et son rendement, sa teneur en huile, son développement et sa morphologie
}

\author{
B Chervet, $F$ Vear * \\ INRA, station d'amélioration des plantes, domaine de Crouelle, 63039 Clermont-Ferrand Cedex, France
}

(Reçu le 8 septembre 1988 ; accepté le 31 octobre 1989)

Résumé - Dans une étude sur 25 hybrides de tournesol, issus d'un plan factoriel de croisement, ainsi que des variétés cultivées, 2 matrices de corrélations linéaires totales entre différents paramètres ont été réalisées afin d'étudier les liaisons entre différents caractères du développement et l'humidité à la récolte, le rendement en grains et la teneur en huile.

La précocité à la récolte est corrélée avec la durée totale levée-maturité physiologique $\left(r=0,74^{\star *}\right)$, et plutôt avec la durée floraison-maturité physiologique $\left(0,69^{* \star}\right)$ que la durée levée-floraison $\left(0,54^{*}\right)$. Les caractères morphologiques ne sont pas révélateurs de la précocité. Le rendement est corrélé avec des caractères morphologiques de vigueur, mais parmi ses composantes le nombre de grains par capitule paraît plus important que le poids de $1000 \mathrm{grains}$. La teneur en huile est peu corrélée avec d'autres caractères.

En l'absence de corrélation rendement-précocité la sélection de tournesols précoces à haut rendement devrait être possible mais nécessiterait des observations en fin de cycle de la maturité des graines.

corrélation linéaire totale / poids de 1000 grains / nombre de grains par capitule / caractère morphologique / floraison / maturité physiologique

Summary - A study of the relations between earliness in sunflowers and yield, oil content, development and morphology. The developmental and agronomic characteristics of sunflowers were observed in trials involving 25 hybrids from a factorial cross and 3 commercial varieties, over 2 years, 1986 and 1987. There were significant differences between genotypes for all characteristics except the duration sowing-emergence (table l). From the results, 2 matrices of total linear correlations were calculated between different parameters in order to study relations between developmental stages and earliness at harvest, yield and oil content (tables II and III).

The length of developmental phases before and after flowering are independent. The duration emergencephysiological maturity was correlated with the periods before and after flowering, but more closely with the duration flowering-maturity $\left(r=0.87^{* *}\right)$. Earliness at harvest appears correlated with the total duration emergencephysiological maturity $\left(r=0.74^{* *}\right)$, and follows the same pattern, being more especially related to the duration flowering-physiological maturity $\left(r=0.69^{* *}\right)$ than to the duration emergence-flowering $(r=0.54 *)$. Of the morphological characters observed, most do not provide any useful indication concerning earliness; only leaf number shows a significant correlation with emergence-flowering and emergence-maturity.

In contrast, yield is significantly correlated with morphological characters which vary with plant vigour, such as head and basal stem diameter and plant height. For the components directly determining yield, seed number per capitulum appears more important than 1000 seed weight. In the conditions of the trials, yield appears most related to characters determined before flowering. Oil content is not closely linked with other characters, except lateness to some extent.

It is concluded that since earliness at harvest depends on the sum of all the vegetative stages, more particularly those after flowering, no juvenile character provides a satisfactory forecast. Earliness can only be determined by endof-cycle observations on seed maturation. However, the absence of any significant correlation between yield and earliness suggests that it should be possible to select for high yielding early sunflowers.

total linear correlation / 1000 seed weight / seed number per capitulum / morphological character / flowering / physiological maturity

\footnotetext{
* Correspondance et tirés à part.
} 


\section{INTRODUCTION}

Les surfaces de tournesol en France ont été multipliées par 30 ces 10 dernières années ( 36000 ha en 1978, 1000000 ha en 1987). L'extension importante de la culture au nord de la Loire pose 2 problèmes : premièrement le cycle végétatif doit être réalisé avec des températures plus faibles, deuxièmement les risques de maladie de fin de cycle (Sclerotinia, Botrytis) sont plus élevés lors d'étés et d'automnes humides. Ces problèmes pourraient être résolus par l'utilisation de variétés ayant un cycle plus court, desséchant plus vite, mais dont le rendement n'est pas trop diminué. Des programmes de sélection ayant la précocité à la récolte comme objectif sont donc nécessaires.

Cependant la précocité, déterminée par l'humidité des graines, est un caractère assez lourd à mesurer. Il faut non seulement suivre les plantes jusqu'à la fin du cycle mais aussi soit disposer d'au moins $50 \mathrm{~g}$ de graines pour permettre des mesures automatisées, ce qui exclut l'utilisation de plantes individuelles, soit réaliser des mesures à l'étuve, coûteuses en temps et en main d'œuvre. C'est pourquoi nous avons entrepris une étude de corrélations afin de déterminer s'il existe 1 ou plusieurs caractères, au cours du développement, ou des paramètres biométriques, permettant de trier pendant un programme de sélection pour la précocité à la récolte. Plus généralement, les relations entre la précocité à la récolte et d'autres caractères pourraient aider à expliquer comment le premier est déterminé. Puisque la précocité doit être associée avec un rendement et une teneur en huile satisfaisants, nous avons, par ailleurs, étudié les relations entre les différents caractères retenus et le rendement en grain ou la teneur en huile.

Si pour les facteurs reliés au rendement en grains et à la teneur en huile chez le tournesol de nombreuses études ont été réalisées (Ross, 1939 ; Putt, 1943 ; Russel, 1953 ; Skoric, 1974 ; Zali et Samadi, 1978 ; Kesteloot et al, 1978) pour l'humidité à la récolte aucune analyse ne semble avoir été publiée jusqu'à présent.

\section{MATÉRIELS ET MÉTHODES}

\section{Matériel}

Le matériel étudié était constitué de 25 génotypes, issus du croisement factoriel de 5 lignées femelles tardives (BL, CX, CG, CD, NG) par 5 lignées mâles précoces (PRS1, RHA 274, PB2, PRS6, ZN41) et de 3 variétés : Frankasol (précoce), Mirasol (mi-précoce), Topflor (tardif).
Le schéma expérimental que nous avons utilisé était de type bloc randomisé, avec des parcelles de $12 \mathrm{~m}^{2}$. En 1986 l'essai était composé de 30 traitements (27 génotypes issus du plan de croisement dont 3 génotypes (CG.PRS6, CG.PB2, CD.PRS1) répétés 2 fois + les 3 variétés) et 27 génotypes en 1987 (25 génotypes issus du plan de croisement + Frankasol + Topflor). La première année, il y avait 2 répétitions dans chacun des 2 lieux : Joze et Les Martres sur Morge (Puy de Dôme). La seconde année les 3 répétitions étaient toutes situées à Pont du Château (Puy de Dôme). Dans tous les cas les essais étaient implantés dans des terres noires de Limagne. Les 2 années, les printemps ont été humides; en 1986 il y a eu une forte sécheresse en août et septembre, en 1987 des températures élevées en septembre (supérieures à $30^{\circ} \mathrm{C}$ ).

Les différents stades de développement du tournesol ont été notés. Ce sont : la levée, l'apparition du bouton floral $\left(E_{1}\right.$ pour le CETIOM, $R_{1}$ pour Schneiter et Miller, 1981), la floraison (un ligule perpendiculaire au plateau du capitule). En 1987 nous avons en plus noté la maturité physiologique (capitule jaune, bractées brunes). Pour chaque génotype un stade phénologique est considéré comme atteint lorsque c'est le cas pour $50 \%$ des plantes. La durée entre chaque stade a été calculée, et en 1987 la somme de températures (base $6^{\circ} \mathrm{C}$ ) correspondante.

Un certain nombre de caractères agronomiques ont été observés ou mesurés : nombre de feuilles, diamètre du capitule à maturité, hauteur à la floraison, diamètre de la tige au collet, rendement en grain sec ( $0 \%$ d'eau) en q/ha, poids de 1000 grains, nombre de grains par capitule. La teneur en huile des graines sèches a été mesurée par résonance magnétique nucléaire (Brucker Minispec 10). Enfin, l'humidité à la récolte a été estimée après étuvage d'un échantillon à $80^{\circ} \mathrm{C}$ pendant $48 \mathrm{~h}$.

\section{RÉSULTATS}

Le tableau I présente un résumé des résultats ; pour toutes les variables sauf la durée semislevée en 1987, les différences génotypiques sont significatives.

Les tableaux II et III présentent les matrices de corrélation linéaires totales pour les 2 études. Les coefficients de corrélations seront notés $r_{1}$ pour les corrélations obtenues en 1986 et $r_{2}$ pour celles obtenues en 1987.

La différence dans les humidités à la récolte entre 1986 et 1987 (tableau 1) résulte d'une plus grande sécheresse en 1987 et d'une récolte plus tardive. Les 2 années représentent la gamme des humidités rencontrées dans les conditions agricoles. Ainsi elles permettent de déterminer si les relations varient en fonction des conditions environnementales. Chervet (1988) a montré des interactions génotypes-années pour les caractères nombre de jours et somme de températures, bouton floral-floraison, nombre de jours levée-floraison, taille, nombre de feuilles, teneur 
Tableau I. Résultats des analyses de variance des différents paramètres étudiés.

\begin{tabular}{|c|c|c|c|c|c|c|c|c|}
\hline & \multicolumn{4}{|c|}{1986} & \multicolumn{4}{|c|}{1987} \\
\hline & moyen & $\min$ & $\max$ & Fgén & moy & $\min$ & $\max$ & F gén \\
\hline $\begin{array}{l}\text { NJ semis-levée } \\
\text { NJ levée-bt flo } \\
\text { NJ bt flo-flor } \\
\text { NJ flor-mat phy } \\
\text { NJ levée-flo } \\
\text { NJ levée-mat phy } \\
\text { som temp levée-bt flor } \\
\text { som temp bt flo-flor } \\
\text { som temp flo-mat phy } \\
\text { hauteur } \\
\text { nombre de feuilles } \\
\text { diamètre du capitule } \\
\text { diamètre au collet } \\
\text { poids de } 1000 \text { grains } \\
\text { nbre grains par capitule } \\
\text { rendement } \\
\text { teneur en huile } \\
\text { humidité à la récolte }\end{array}$ & $\begin{array}{c}163,7 \\
28,6 \\
16,7 \\
2,34 \\
47,7 \\
850,2 \\
24,2 \\
48,6 \\
25,1\end{array}$ & $\begin{array}{c}145,0 \\
24,5 \\
15,3 \\
2,14 \\
39,1 \\
633,5 \\
18,8 \\
41,2 \\
15,7\end{array}$ & $\begin{array}{c}182,0 \\
33,0 \\
20,4 \\
2,97 \\
58,5 \\
1244,3 \\
34,4 \\
55,6 \\
34,8\end{array}$ & $\begin{array}{r}6,27^{\star \star} \\
19,31^{\star \star} \\
4,85^{\star *} \\
2,77^{\star \star} \\
9,18^{\star *} \\
1,88^{*} \\
1,78^{*} \\
25,35^{\star \star} \\
8,11^{* *}\end{array}$ & $\begin{array}{c}10,7 \\
47,8 \\
27,4 \\
50,9 \\
75,3 \\
126,3 \\
346 \\
348 \\
692 \\
179,5 \\
27,2\end{array}$ & $\begin{array}{c}10,3 \\
42,0 \\
24,6 \\
46,0 \\
71,3 \\
118,0 \\
302 \\
316 \\
632 \\
143,0 \\
23,4\end{array}$ & $\begin{array}{c}11,3 \\
52,7 \\
31,0 \\
56,0 \\
82,6 \\
138,7 \\
358 \\
421 \\
789 \\
202,1 \\
31,1\end{array}$ & $\begin{array}{r}5,58 \text { NS } \\
8,07^{* *} \\
3,27^{* *} \\
19,08^{* *} \\
15,59^{* *} \\
12,67^{* *} \\
9,30^{\star *} \\
3,83^{\star *} \\
6,55^{* *} \\
7,59^{* *} \\
44,31^{* *}\end{array}$ \\
\hline
\end{tabular}

Tableau II. Corrélations linéaires totales entre 13 variables (1986).

Coeff. de corrólation (28 ddl) : $r_{0,05}=0,36, r_{0,01}=0,46$

dicap : diamètre du capitule; haute : hauteur ; nfeuil : nombre de feuilles ; rdt : rendement; ngcap : nombre de grains par capitule; pmg : poids de 1000 grains ; hurec : humidité à la récolte ; njsle : nombre de jours semis-levée ; njlbf : nombre de jours levée-bouton floral ; njbff : nombre de jours bouton floral-floraison ; njfft : nombre de jours levée-floraison ; dicol : diamètre au collet.

\begin{tabular}{|c|c|c|c|c|c|c|c|c|c|c|c|c|c|}
\hline & dicap & haute & nfeui & $r d t$ & ngcap & $p m g$ & hurec & njsle & nilbf & nibff & njlfi & dicol & huile \\
\hline dicap & 1,00 & & & & & & & & & & & & \\
\hline haute & 0,21 & 1,00 & & & & & & & & & & & \\
\hline nfeui & $-0,19$ & 0,24 & 1,00 & & & & & & & & & & \\
\hline$r d t$ & 0,52 & 0,14 & $-0,08$ & 1,00 & & & & & & & & & \\
\hline ngcap & 0,15 & 0,19 & 0,21 & 0,80 & 1,00 & & & & & & & & \\
\hline pmg & 0,61 & $-0,12$ & $-0,44$ & 0,45 & $-0,18$ & 1,00 & & & & & & & \\
\hline hurec & 0,16 & $-0,31$ & 0,28 & 0,07 & $-0,10$ & 0,31 & 1,00 & & & & & & \\
\hline nisle & $-0,19$ & 0,12 & 0,17 & $-0,35$ & $-0,27$ & $-0,21$ & $-0,19$ & 1,00 & & & & & \\
\hline njlbf & 0,13 & 0,41 & 0,47 & 0,10 & 0,28 & $-0,22$ & 0,41 & $-0,20$ & 1,00 & & & & \\
\hline njbff & $-0,46$ & $-0,59$ & 0,01 & $-0,15$ & 0,03 & $-0,27$ & 0,16 & $-0,08$ & $-0,39$ & 1,00 & & & \\
\hline nilfl & $-0,28$ & $-0,08$ & 0,48 & $-0,10$ & 0,23 & $-0,48$ & 0,54 & $-0,24$ & 0,65 & 0,42 & 1,00 & & \\
\hline dicol & 0,70 & 0,34 & $-0,06$ & 0,61 & 0,41 & 0,33 & 0,02 & 0,05 & 0,11 & $-0,43$ & $-0,27$ & 1,00 & \\
\hline huile & $-0,54$ & $-0,25$ & 0,06 & $-0,47$ & $-0,0$ & $-0,63$ & 0,07 & 0,02 & 0,03 & 0,61 & 0,54 & $-0,57$ & 1,00 \\
\hline
\end{tabular}

en huile et humidité à la récolte. Cependant, on peut remarquer dans les tableaux II et III que pour les caractères observés les 2 années, les coefficients de corrélation, significatifs ou non, sont souvent semblables et, ainsi, il paraît intéressant de les étudier en même temps.

\section{Précocité}

\section{Durée de phases}

Pendant la première moitié du cycle, la durée levée-floraison est corrélée, les 2 années, avec la durée levée-bouton floral $\left(r_{1}=0,65^{\star \star}\right.$, $\left.r_{2}=0,73^{\star \star}\right)$, mais seulement en 1986 avec le nombre de jours bouton floral-floraison $\left(r_{1}=0,42 *\right)$. Ce résultat peut être relié avec la différence des durées moyennes des deux phases, 45,2 j pour levée-bouton floral et seulement 27,4 j pour bouton floral-floraison. La deuxième partie du cycle, floraison-maturité physiologique, apparaît indépendante de la première car il n'y a pas de corrélation entre les durées levée-floraison et floraison-maturité physiologique. La durée levée-maturité physiologique, soit la longueur totale du cycle, est corrélée avec toutes ses composantes mais il faut noter que le coefficient le plus élevé apparaît pour le nombre de jours floraison-maturité physiologique 
Tableau III. Corrélations linéaires totales entre 15 variables (1987).

Coeff. de corrélation $(25 \mathrm{ddl}): r_{0.05}=0,38, r_{0,01}=0,49$

njfmp : nombre de jours floraison-maturité physiologique ; njlmp = nombre de jours levée-maturité physiologique ; stlbf : somme des températures levéebouton floral ; stbff : somme des températures bouton floral-floraison ; stfmp : somme des températures floraison-maturité physiologique.

\begin{tabular}{|c|c|c|c|c|c|c|c|c|c|c|c|c|c|c|}
\hline & njlbf & $n j b f f$ & njfmp & $p m g$ & ngcap & $r d t$ & hurec & nfeui & haute & njlfl & nilmp & huile & $s t / b f$ & stbff stfmp \\
\hline nilbf & 1,00 & & & & & & & & & & & & & \\
\hline nibff & $-0,53$ & 1,00 & & & & & & & & & & & & \\
\hline nifmp & 0,38 & $-0,18$ & 1,00 & & & & & & & & & & & \\
\hline$p m g$ & $-0,21$ & $-0,14$ & 0,44 & 1,00 & & & & & & & & & & \\
\hline ngcap & 0,44 & 0,09 & $-0,32$ & $-0,73$ & 1,00 & & & & & & & & & \\
\hline$r d t$ & 0,49 & $-0,02$ & $-0,11$ & $-0,24$ & 0,82 & 1,00 & & & & & & & & \\
\hline hurec & 0,45 & $-0,10$ & 0,69 & 0,21 & 0,12 & 0,00 & 1,00 & & & & & & & \\
\hline nfeui & 0,49 & 0,01 & 0,27 & $-0,20$ & 0,37 & 0,39 & 0,37 & 1,00 & & & & & & \\
\hline haute & 0,58 & $-0,08$ & 0,10 & $-0,37$ & 0,56 & 0,50 & 0,23 & 0,39 & 1,00 & & & & & \\
\hline njlfl & 0,73 & 0,18 & 0,36 & $-0,30$ & 0,56 & 0,53 & 0,46 & 0,52 & 0,62 & 1,00 & & & & \\
\hline njlmp & 0,63 & $-0,02$ & 0,87 & 0,17 & 0,05 & 0,22 & 0,74 & 0,52 & 0,37 & 0,74 & 1,00 & & & \\
\hline huile & 0,09 & 0,15 & 0,25 & $-0,12$ & 0,08 & 0,05 & 0,16 & 0,27 & 0,14 & 0,21 & $-0,43$ & 1,00 & & \\
\hline stlbf & 0,99 & $-0,54$ & 0,33 & $-0,23$ & 0,43 & 0,46 & 0,40 & 0,44 & 0,56 & 0,71 & 0,59 & 0,54 & 1,00 & \\
\hline stbff & 0,03 & 0,62 & 0,04 & $-0,21$ & 0,30 & 0,27 & 0,41 & 0,27 & 0,29 & 0,52 & 0,32 & 0,03 & $-0,01$ & 1,00 \\
\hline stfmp & 0,35 & $-0,07$ & 0,86 & 0,37 & $-0,25$ & $-0,06$ & 0,71 & 0,52 & 0,09 & 0,34 & 0,85 & 0,26 & 0,31 & $0,11 \quad 1,00$ \\
\hline
\end{tabular}

$\left(r_{2}=0,87^{\star \star}\right)$. Les corrélations avec les durées levée-floraison $\left(r_{2}=0,74^{* \star}\right)$, et levée-bouton floral $\left(r_{2}=0,63^{\star *}\right)$, ainsi qu'avec les sommes des températures correspondantes sont néanmoins hautement significatives. Parmi les caractères morphologiques étudiés, seul le nombre de feuilles peut être relié avec les durées levée-floraison et levée-maturité physiologique (respectivement $r_{1}=0,48^{\star \star}, r_{2}=0,52^{\star \star}$ et $\left.r_{2}=0,52^{\star \star}\right)$.

\section{Humidité à la récolte}

Les meilleurs prédicteurs de ce paramètre sont le nombre de jours levée-maturité physiologique $\left(r_{2}=0,74^{* *}\right)$, la somme des températures entre la floraison et la maturité physiologique $\left(r_{2}=0,70^{\star \star}\right)$, et la durée de cette même période $\left(0,69^{* *}\right)$. L'humidité à la récolte est reliée, mais moins étroitement, au nombre de jours levéebouton floral $\left(r_{1}=0,41^{*}, \quad r_{2}=0,45^{*}\right)$. Ainsi comme pour la durée du cycle jusqu'à maturité physiologique, la précocité à la récolte parait liée avec les durées des différentes phases mais surtout avec le comportement postfloral.

\section{Rendement}

Dans l'essai de 1987 le rendement est corrélé avec le nombre de jours levée-bouton floral $\left(r_{2}=0,49^{\star \star}\right)$ et le nombre de jours levée-floraison $\left(r_{2}=0,53^{* *}\right)$ mais semble indépendant de la durée levée-maturité physiologique. Peut-être est-ce dû à un effet climatique, la sécheresse d'août, donc après la floraison, défavorisant les génotypes tardifs à la récolte. Contrairement à la précocité, le rendement peut être relié avec un certain nombre de caractères morphologiques de la plante : diamètre du capitule à maturité $\left(r_{1}=0,52^{* *}\right)$, diamètre au collet $\left(r_{1}=0,61^{* *}\right)$ en 1986, et hauteur à la floraison en 1987 $\left(r_{2}=0,50^{* *}\right)$. Tous ces paramètres traduisent une bonne vigueur de la plante.

Parmi les composantes du rendement, la plus importante est le nombre de grains par capitule $\left(r_{1}=0,80^{* *}, r_{2}=0,82^{\star *}\right)$. En 1986 le rendement et le poids de 1000 grains sont liés $\left(r_{1}=0,45^{*}\right)$ alors qu'il ne le sont pas en 1987. Le nombre de grains par capitule et le poids de 1000 grains montrent une corrélation négative significative $\left(r_{2}=-0,73\right)$. Quand on examine les corrélations entre ces 2 composantes et les caractères de la morphologie et du développement, on trouve des relations différentes. Le nombre de grains par capitule est corrélé avec le diamètre au collet $\left(r_{1}=0,41^{*}\right)$, la hauteur $\left(r_{2}=0,56^{* *}\right)$, les durées levée-floraison $\left(r_{2}=0,56^{* *}\right)$ et levée-bouton floral $\left(r_{2}=0,44^{*}\right)$. Au contraire, le poids de 1000 grains est corrélé avec le diamètre final du capitule qui augmente surtout à partir du début de la fécondation des graines. Ainsi le nombre de grains est relié à une vigueur avant floraison, le poids de 1000 grains avec une vigueur après floraison. Les conditions de sécheresse postflorale ayant probablement limité le métabolisme, le rendement est surtout déterminé par le nombre de grains et la vigueur de la plante au moment de la mise en place de ce caractère.

\section{Teneur en huile}

On n'observe qu'en 1986 des corrélations significatives entre la teneur en huile et d'autres paramètres. Certaines sont positives : nombre de jours bouton floral-floraison $\left(r_{1}=0,61^{* \star}\right)$, 
nombre de jours levée-floraison $\left(r_{1}=0,54^{\star \star}\right)$, d'autres sont négatives : poids de 1000 grains $\left(r_{1}=0,63^{\star \star}\right)$, diamètre au collet $\left(r_{1}=0,57^{\star \star}\right)$, diamètre du capitule $\left(-0,54^{\star \star}\right)$. II semble donc que les génotypes tardifs à la floraison aient tendance à être plus riches en huile, mais la synthèse de l'huile est un mécanisme particulier et ne dépend pas de la vigueur générale de la plante. La corrélation négative entre la teneur en huile et le rendement en grains en $1986\left(r=-0,47^{* *}\right)$ confirme l'indépendance par rapport aux caractéristiques de vigueur. Par ailleurs, pour les mêmes données, il n'y a aucune corrélation entre la teneur en huile et le rendement en huile $(r=0,04)$.

\section{DISCUSSION}

Ces études portent sur des corrélations phénotypiques, qui ne sont pas toujours de bons prédicteurs des corrélations génétiques. Néanmoins, elles permettent de distinguer les tendances, et ainsi de déterminer les caractères importants dans un programme de sélection pour la précocité, et donc méritant des études approfondies d'héritabilité.

\section{Durée de cycle, précocité}

Sur le matériel que nous avons étudié, assez représentatif des génotypes de tournesol cultivés ou utilisés en sélection, les différentes phases de développement paraissent indépendantes, une phase courte ou longue n'étant pas indicative de la durée des autres phases. La maturité physiologique et la précocité à la récolte semblent dépendre de l'ensemble des phases mais surtout de la durée floraison-maturité physiologique, mesurée en jours ou en somme de températures. Le déroulement de la maturation et du dessèchement des graines et du capitule est spécifique selon les génotypes (Chervet et Vear, 1989). Les différences de comportement après floraison sont peut-être plus grandes qu'avant floraison, car pendant ce dernier stade les variétés précoce (Frankasol) et tardive (Topflor) se différencient seulement par la durée de la phase levée-bouton floral (Chervet, 1988).

Ces études sur hybrides apportent des résultats comparables à ceux de Burlov et Redkov (1983) sur 6 lignées. Les auteurs ont montré que la période de croissance était corrélée avec les durées levée-bouton floral et floraison-maturité physiologique. Ils ont trouvé, comme nous, que le nombre de feuilles était corrélé avec la durée levée-bouton floral. Cette relation pourrait s'expli- quer par un besoin assez constant de somme de températures, ou de temps, pour le développement de chaque feuille. Ce caractère nous a paru le seul relié avec les stades de développement, contrairement à Russel (1953) qui a trouvé que la surface foliaire, la hauteur, la date de floraison, le diamètre de la tige et du capitule sont liés à la date de maturité. En dehors du diamètre du capitule les caractères observés sont mesurés avant floraison et ainsi ne prennent pas en compte le déroulement de la maturation. On pourrait penser qu'il serait plus intéressant d'examiner les caractères morphologiques après la floraison, tels que le nombre de feuilles sèches ou l'aspect du capitule. Néanmoins la maturation des graines est indépendante de celle du capitule (Chervet et Vear, 1989); ainsi l'évolution des graines peut être différente de celle de la plante mère.

\section{Rendement}

Le rendement du tournesol a déjà été largement analysé et la présente étude montrant qu'il est corrélé avec le nombre de grains par capitule et peut l'être avec le poids de 1000 grains confirme les travaux de Podtar et al (1977), Kesteloot et al (1978), et Merrien et al (1982). Certains auteurs ont trouvé une corrélation uniquement avec le poids de 1000 grains (Sidhu et Bains, 1980) tandis que les résultats rapportés ici font apparaitre le nombre de grains par capitule comme étant la composante la plus importante du rendement. La différence pourrait résulter des conditions climatiques dans lesquelles ont été réalisés nos essais : les printemps humides ayant permis l'expression des potentiels de nombre de grains, tandis que la sécheresse estivale aurait limité leur remplissage. La corrélation négative nombre de grains-poids de 1000 grains pourrait être le résultat du partage d'une même quantité d'assimilats dans un nombre de grains variable. Néanmoins il serait nécessaire de réaliser une étude avec irrigation afin de s'assurer que les conditions d'alimentation en eau n'influencent pas les résultats.

Les caractères morphologiques apparaissent plus directement liés au rendement qu'à la précocité. Putt (1943) a observé une relation entre le rendement et la hauteur, les diamètres de la tige et du capitule, Pathak (1974) et Merrien et al (1982) entre le rendement et ces 2 derniers paramètres, Burns (1970), Ayyasami et al (1977), et Vannozzi et al (1986) avec le diamètre du capitule, enfin Moradi et Vojdani (1974) et Podtar et al (1977) avec la surface foliaire. Ce sont tous des caractères de vigueur. 


\section{Teneur en huile}

II n'apparaît pas de liaison étroite entre la teneur en huile et d'autres caractères. Dans le matériel étudié, il y avait une relation négative avec le rendement en graines et les paramètres de vigueur qui y sont associés. Dans la littérature (Putt, 1943, Skoric, 1974, Moradi et Vojdani, 1974, Zali et Samadi, 1978) il existe des résultats discordants. Ces différences pourraient être dues aux origines génétiques du matériel sans être des liaisons physiologiques.

Néanmoins la liaison négative avec le rendement et le poids de 1000 grains que nous avons observée, déjà mise en évidence par Putt (1943) et dans une étude sur une centaine de lignées (Vear, non publié), pose la question suivante : est-ce qu'une très haute teneur en huile, demandant plus d'énergie que la même quantité de cellulose, ne conduit pas à une plus faible production des matières "non-huile" dans la graine, et donc à des graines plus petites et à une baisse de rendement ? L'absence de corrélation entre la teneur en huile et le rendement en huile appuie cette hypothèse.

En conclusion, il apparaît dans les conditions de notre étude que les durées des phases de développement du tournesol sont indépendantes et que la précocité de récolte est fonction de l'ensemble, et ne peut pas être expliquée de façon satisfaisante par des caractères juvéniles du développement ou de la morphologie.

Comme Kesteloot et al (1978), nous n'avons pas trouvé dans notre étude de corrélation entre l'humidité à la récolte et le rendement. Ceci nous encourage à penser qu'un programme de sélection peut permettre un gain de précocité sans diminution inévitable du rendement, bien que d'autres travaux soient nécessaires pour mieux connaître la relation entre ces 2 caractères. Chervet (1988) a estimé I'héritabilité au sens large de l'humidité à la récolte à environ $60 \%$. Afin d'entreprendre efficacement un programme de sélection pour la précocité de maturité, il sera important de confirmer cette estimation, car les présents résultats indiquent que les mesures de précocité doivent obligatoirement être faites sur les graines au cours de leur maturation et dessèchement.

\section{RÉFÉRENCES}

Ayyasamy MK, Krishnaraju J, Sivasankaran D (1977) Biometric studies in sunflower. Madras Agric $J, 64$, $7,430-432$
Burlov VV, Redkov VV (1983) Correlation analysis of some economic characters determining growth period duration and yield in sunflower. Plant Breed Abst, 53, 8, 619-620

Burns RE (1970) Head size of sunflower as an indicator of plot yield. Agron J, 62, 112-113

Chervet B (1988) Étude agronomique et physiologique de la précocité chez le tournesol. Thèse d'université, Clermont-Ferrand, $147 \mathrm{p}$

Chervet B, Vear F (1989) Évolution des caractéristiques de la graine et du capitule chez le tournesol au cours de la maturation. Agronomie, 9, 305-313

Kesteloot JA, Leclercq P, Serieys H (1978) Recherche contribuant à la détermination des éléments permettant la différenciation entre hybrides à haut et bas rendement dans le tournesol (Helianthus annuus L). 8th Intern Sunflower Conf, Minneapolis, USA, 343-347

Merrien A, Blachet R, Gelfi N, Rellier JP, Rollier M (1982) Voies d'élaboration du rendement chez le tournesol sous différents stress hydriques. $10^{\circ}$ Conf Intern Tournesol, Surfers Paradise, 11-14

Moradi A, Vojdani P (1974) Study of the relationship between leaf surface, grain yield and oil content in different varieties of sunflower. 6th Intern Sunflower Conf, Bucarest, 189-196

Pathak RS (1974) Yield conponents in sunflower. 6th Intern Sunflower Conf, Bucarest, 271-281

Podtar MV, Pawar KR, Sreenivas L (1977) Simple correlation and regression studies between grain yield and yield attributing characters in sunflower. Ind $J$ Agron, 22, 115-116

Putt ED (1943) Association of seed yield and oil content with other characters in sunflower. Sci Agric, 37, 377-383

Ross AM (1939) Some morphological characters of Helianthus annuus $\mathrm{L}$, and their relation to the yield of seed and oil. Sci Agric, 19, 372-379

Russel WA (1953) A study of inter-relationships of seed yield, oil content, and other agronomic characters with sunflower inbred lines and their top crosses. Can J Agric Sci, 33, 291-314

Schneiter AA, Miller JF (1981) Description of sunflower growth stage. Crop Sci, 21, 901-903

Sidhu BS, Bains DS (1980) Correlation between seed yield, yield attributing and quality characteristics of sunflower. Ind J Agron, 25, 1, 156-157

Skoric $D(1974)$ Correlation among the most important characters of sunflower in $\mathbf{R}_{1}$ generation. 6 th Intern Sunflower Conf, Bucarest, 283-289

Vannozzi GP, Belloni P, Martorana F (1986) Correlazione tra fattori produttivi nel girasole (Helianthus annuus L). Sementi Eletta, 32, 25-31

Zali AA, Samadi BY (1978) Association of seed yield and seed oil content with other plant and seed characteristics in Helianthus annuus L. 8th Intern Sunflower Conf, Minneapolis, 164-171 